Molecules 2004, 9, 541-549

molecules

ISSN 1420-3049

http://www.mdpi.org

\title{
Isomerization Reactions of Allylic Alcohols into Ketones with the Grubbs Reagent
}

\section{Katsuyuki Nakashima, Sanae Okamoto, Masakazu Sono and Motoo Tori*}

Faculty of Pharmaceutical Sciences, Tokushima Bunri University, Yamashiro-cho, Tokushima, 770-8514, Japan. Tel. (+81) 88-622-9611, Fax (+81) 88-655-3051.

* Author to whom correspondence should be addressed; e-mail: tori@ph.bunri-u.ac.jp; homepage: http://p.bunri-u.ac.jp/ tori/english/e-tori.html

Received: 26 December 2003; in revised form: 24 March 2004 / Accepted: 3 April 2004 / Published: 30 June 2004

\begin{abstract}
Allylic alcohols were isomerized into ketones by the action of the Grubbs reagent. Some model alcohols were prepared and tested under similar conditions to reveal that less substituted alkenes rearrange more easily. More hindered alcohols are stable under these conditions, however, the simple allylic alcohols tend to isomerize producing ethyl ketone and the corresponding degraded methyl ketone.
\end{abstract}

Keywords: Grubbs reagent, allylic alcohol, isomerization, ketone.

\section{Introduction}

We have previously found and reported that the allylic alcohol $\mathbf{1}$ rearranged into the ketones $\mathbf{2}$ and 3 under ring closing olefin metathesis reaction (RCM) conditions using the Grubbs reagent [Ru] (Scheme 1) [1]. The expected 10-membered carbocycle was not formed, but rather the allylic alcohol 1 isomerized to give ketone 2 as well as the one-carbon degraded ketone 3 in a ratio of 2:1 in 68\% yield. The formation of the one-carbon degraded methyl ketone had been recorded by Hoye and Zhau in 1999 [2] and the mechanism shown to be as depicted in Scheme 2. The Grubbs reagent attacks compound A to produce another carbene complex B, which rearranges into enol C. Then, 
tautomerization to $\mathrm{D}$ and reductive elimination affords the degraded ketone E. However, our observation of the formation of the isomerized ketone $\mathbf{2}$ was the first example, although some isomerization reactions using $\mathrm{Ru}$ complexes had been reported [3-5]. We became interested in the scope of these reactions and herein we report the results of the reaction of some different allylic alcohols with the Grubbs reagent.

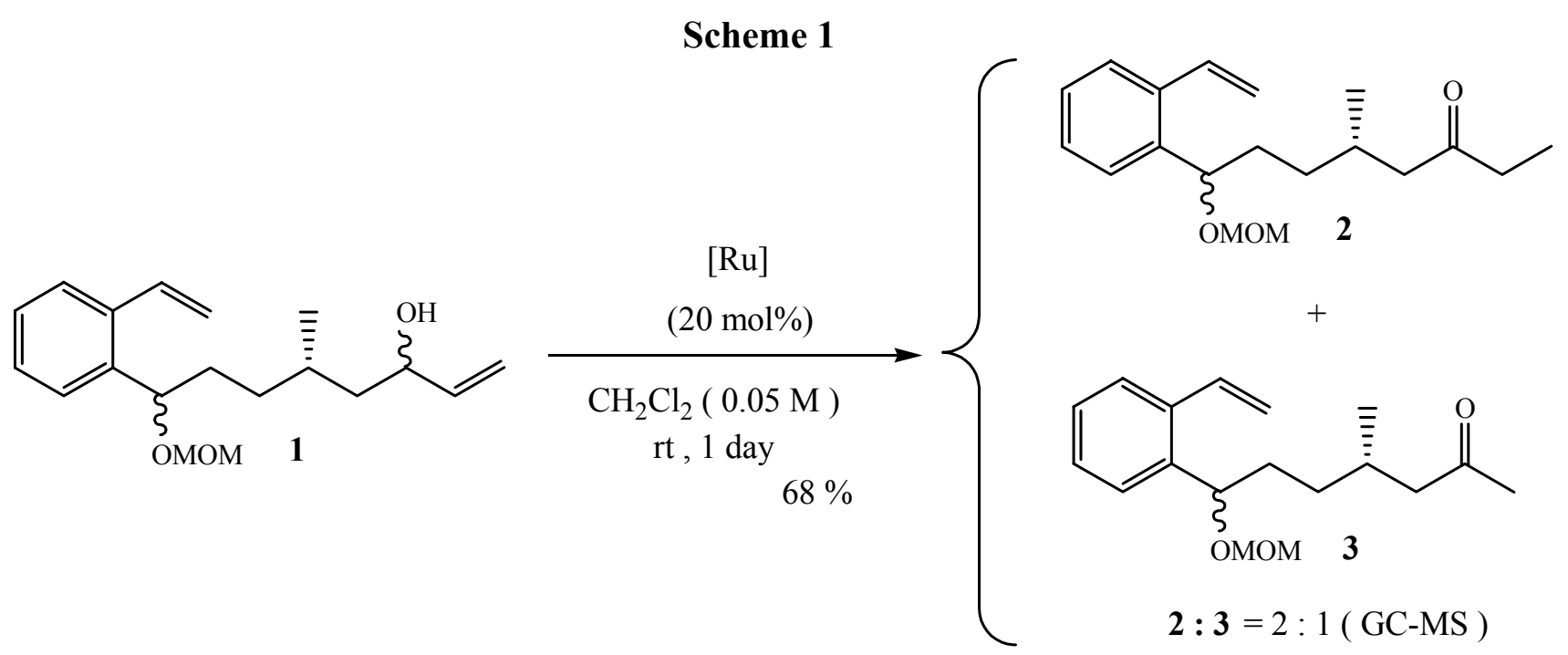

Scheme 2<smiles>C=C[C@@H](O)[C@@H](C)[C@H](O)[C@H](C)CO[13CH3]</smiles>

A

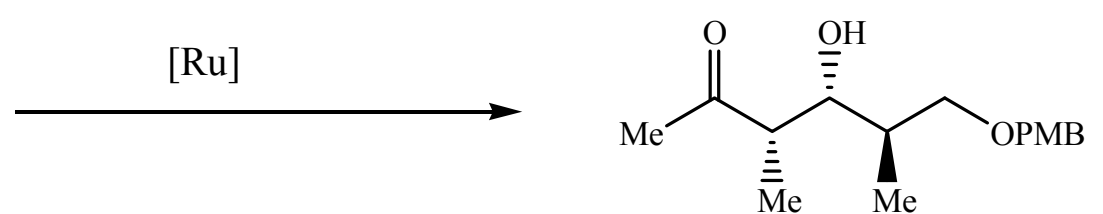

$\mathrm{E}$

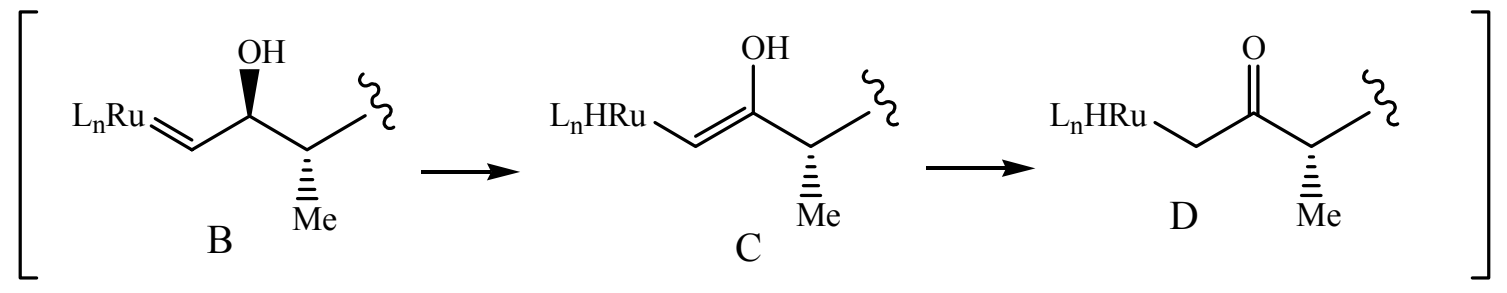

\section{Results and Discussion}

Mono-protected butanediol 4 [6] was oxidized into aldehyde 5 [6], which was alkylated as shown in Scheme 3 to afford three kinds of alcohols 6, 7 and 8 . Compound 6 was treated with the Grubbs reagent in dichloromethane (Scheme 4). As seen in entry 1, most of the starting material remained unchanged, but a small amount of ketone 10 [7] was formed. However, when $10 \mathrm{~mol} \%$ of the catalyst was used (entry 2), the starting material was consumed completely and ketones 9 and 10 [7] were 
formed in a ratio of 3:1. Use of more of the catalyst (20 mol \%, entry 3) resulted in a slight change in the ratio of 9 and $\mathbf{1 0}$ [7]. Raising the temperature did not affect the reaction favourably, and instead the starting material remained unchanged (entries 4 and 5). Structures 9 and 10 [7] were easily determined from the spectroscopic data (See Experimental).

Scheme 3

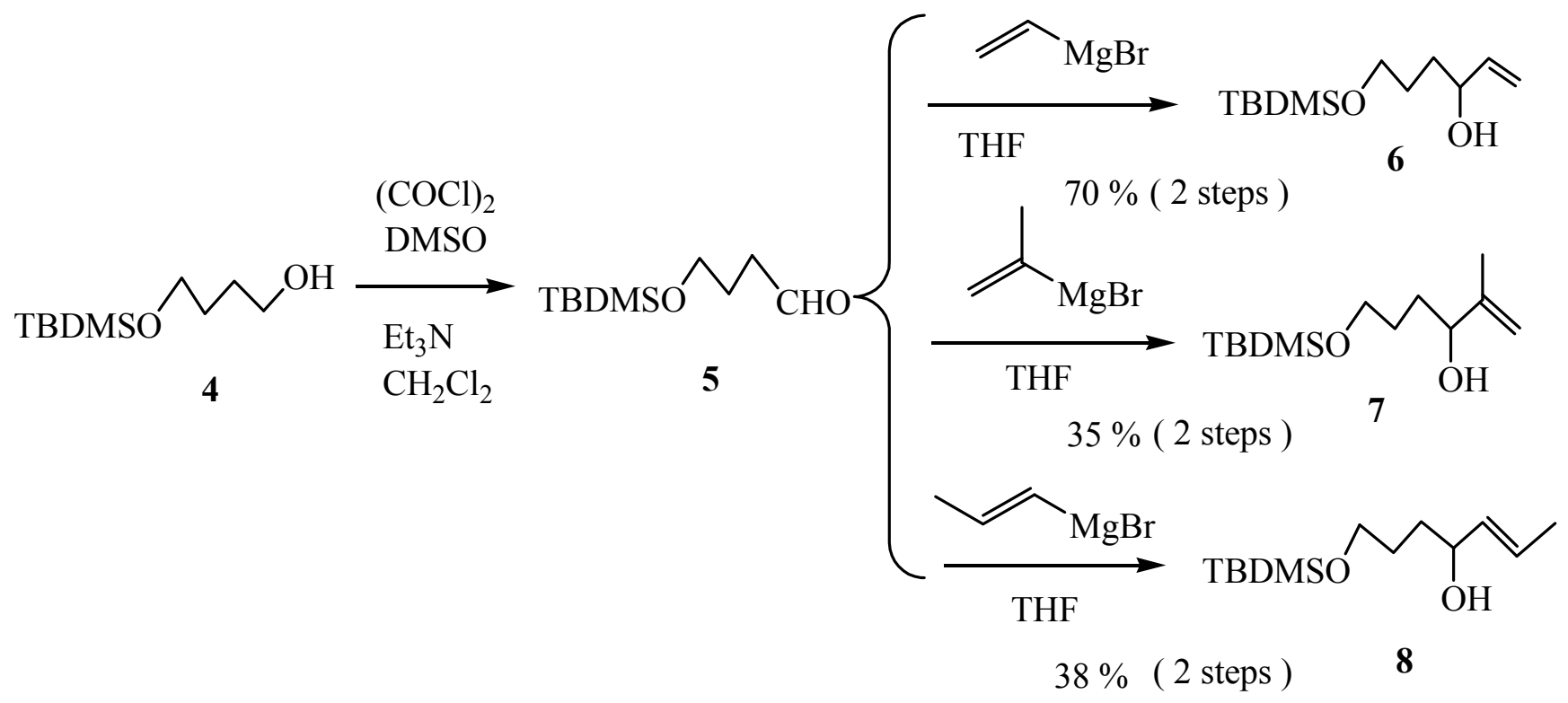

Scheme 4

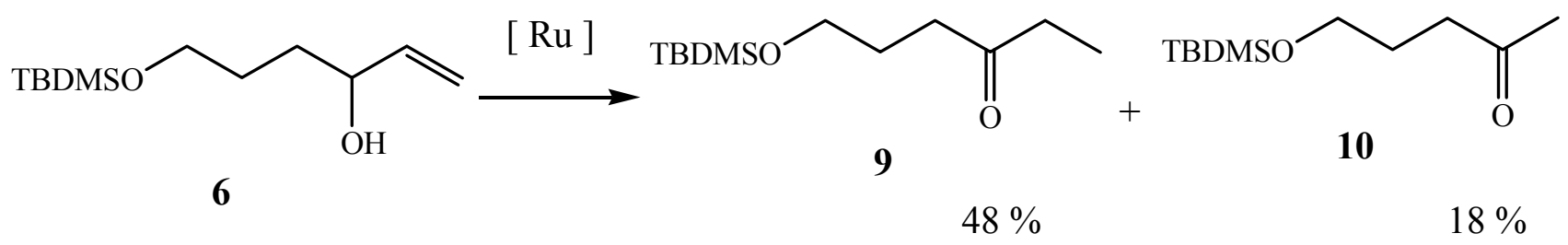

\begin{tabular}{ccccccc}
\hline entry & mol \% & solvent & conc. $(\mathrm{mM})$ & temp. $\left({ }^{\circ} \mathrm{C}\right)$ & time (days) & results \\
\hline 1 & 5 & $\mathrm{CH}_{2} \mathrm{Cl}_{2}$ & 10 & $\mathrm{rt}$ & 1 & $26: 9: \mathbf{1 0}$ \\
2 & 10 & $\mathrm{CH}_{2} \mathrm{Cl}_{2}$ & 10 & $\mathrm{rt}$ & 1 & $0: 3: 1$ \\
3 & 20 & $\mathrm{CH}_{2} \mathrm{Cl}_{2}$ & 10 & $\mathrm{rt}$ & 1 & $0: 2: 1$ \\
4 & 5 & $\mathrm{CH}_{2} \mathrm{Cl}_{2}$ & 10 & 40 & 1 & $30: 0: 1$ \\
5 & 10 & $\mathrm{CH}_{2} \mathrm{Cl}_{2}$ & 10 & 40 & 1 & $9: 0: 1$ \\
\hline
\end{tabular}


Next, compound 7 was treated with the Grubbs reagent (initially $20 \mathrm{~mol} \%$ ) in dichloromethane at room temperature (rt) overnight (Scheme 5). Then, $10 \mathrm{~mol} \%$ of the catalyst was added and the mixture stirred overnight. The starting material was recovered after 2 days (entry 1). If the concentration of the reaction mixture was raised to $0.1 \mathrm{M}(20 \mathrm{~mol} \%$ of the catalyst), less than $10 \%$ of the isomerized ketone 9 was produced (entry 2). In the case of alcohol 8 (Scheme 6), the positional isomer of 7 with regards to the methyl group, ketones $\mathbf{1 0}$ and $\mathbf{1 1}$ were formed in a ratio of 1:1 after 4 days but only in minute amounts. These results indicate that the substituents on the double bond affect the ease of the isomerization reaction. Thus, the methyl group substituted at the $\alpha$-position of the hydroxyl group, as in compound 7, interferes with the isomerization reaction, compared to the case of compound 6. On the other hand, compound $\mathbf{8}$ seems more reactive under the conditions used in this study.

Scheme 5

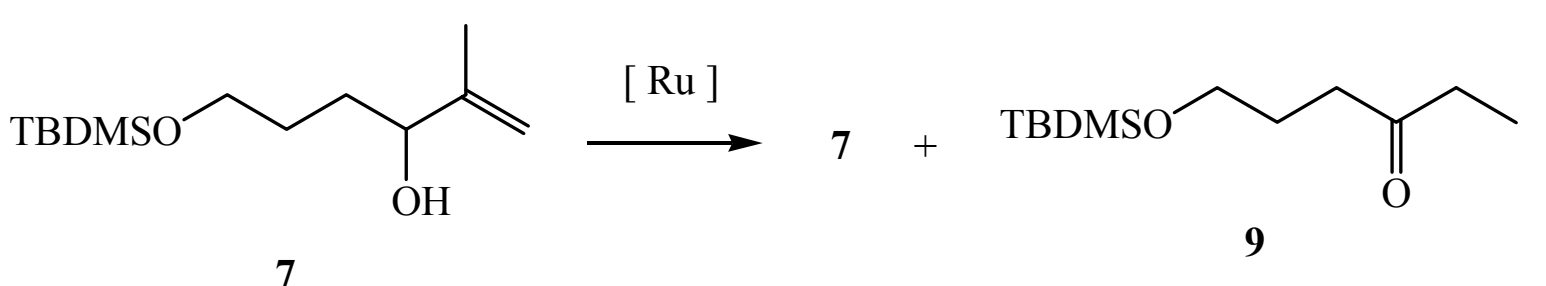

7

\begin{tabular}{ccccccl}
\hline entry & mol \% & solvent & conc. $(\mathrm{mM})$ & temp. $\left({ }^{\circ} \mathrm{C}\right)$ & time (days) & results \\
\hline 1 & $20+10$ & $\mathrm{CH}_{2} \mathrm{Cl}_{2}$ & 10 & $\mathrm{rt}$ & $1+1$ & No Reaction \\
2 & 20 & $\mathrm{CH}_{2} \mathrm{Cl}_{2}$ & 100 & $\mathrm{rt}$ & 1 & $7: 9=28: 3$ \\
\hline
\end{tabular}

\section{Scheme 6}

$[\mathrm{Ru}]$

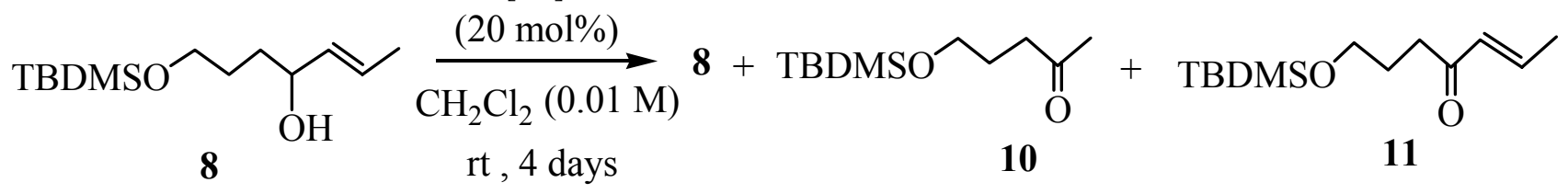

$10: 11=1: 1(\mathrm{GC}-\mathrm{MS})$

Finally we have prepared compound 15, with two methyl groups on the double bond in the $\beta$-position with regards to the hydroxyl group (Scheme 7). In this case the starting material was recovered after 4 days of reaction with the Grubbs reagent (20 or $30 \mathrm{~mol} \%)$ (entries 1-3). After three weeks, oxidation product 16 and dehydration product 17 were produced, in yields of 1 and $47 \%$, respectively. From the above results, this is reasonable, because compound $\mathbf{1 5}$ is much more sterically hindered than compounds 7 and $\mathbf{8}$. 


\section{Scheme 7}
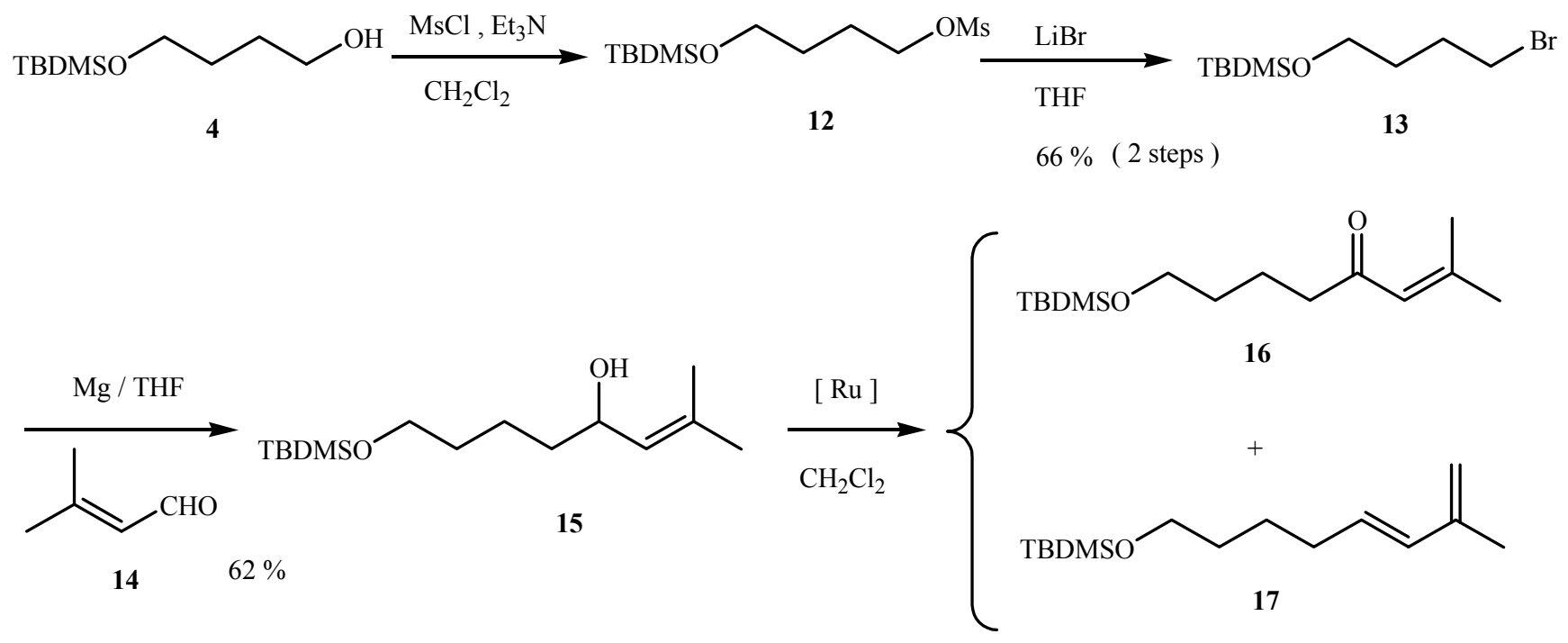

\begin{tabular}{|c|c|c|c|c|c|c|}
\hline entry & mo $1 \%$ & conc. $(\mathrm{mM})$ & temp. $\left({ }^{\circ} \mathrm{C}\right)$ & time (days) & recovery $(\%)$ & yield ( \% ) \\
\hline 1 & 20 & 10 & $\mathrm{rt}$ & 4 & 59 & - \\
\hline 2 & 20 & 100 & 40 & 2 & 100 & - \\
\hline 3 & $20+10$ & 10 & 40 & $2+2$ & 78 & - \\
\hline 4 & $5+5+5+5$ & 10 & $\mathrm{rt}$ & 21 & 8 & $\mathbf{1 6}(1) \quad 17(47)$ \\
\hline
\end{tabular}

\section{Conclusions}

The Grubbs catalyst attacks the terminal alkenes as shown in Scheme 2 to form a carbene complex. Then, rearrangement and reductive elimination produce the degraded ketone. Simple isomerization may occur by coordination of the alkene to the ruthenium complex formed in situ and results in isomerization, although the details are not clear at this stage. The ethyl ketone 9 appears after the methyl ketone $\mathbf{1 0}$ is formed. Therefore, it is reasonable to conclude that some ruthenium species formed in the reaction may be responsible for the formation of the ethyl ketone. Consequently, when planning a RCM reaction, it would appear that the allylic alcohol is not a promising substrate, but rather the use of TES- or TMS-protected compounds is strongly recommended, because some of the side reactions described here may cause problems.

\section{Acknowledgements}

We thank Ms. Yasuko Okamoto (our university) for measurement of the MS spectra. 


\section{Experimental}

\section{General}

The IR spectra were measured with a JASCO FT/IR-500 spectrophotometer. The ${ }^{1} \mathrm{H}$ - and ${ }^{13} \mathrm{C}-\mathrm{NMR}$ spectra were recorded on a Varian Unity 200 or a Varian Mercury 300 spectrometer. Deuteriochloroform was used for NMR and chemical shifts are expressed in ppm and the coupling constants in Hz. The mass spectra including high-resolution mass spectra were taken with a JEOL AX-500 spectrometer. GC-MS was carried out on a HP MS-5973 with GC HP-6890 system. Silica gel BW-300 (200-400 mesh, Fuji silycia) was used for column chromatography, and silica gel 60F 254 plate (0.25 mm, Merck) were used for TLC. All reactions were carried out under an argon atmosphere. THF was distilled from $\mathrm{LiAlH}_{4}$ and then from Na-benzophenone prior to use. The Grubbs reagent was purchased from Strem and used as received. The reagent was weighed in the dry box and was used without purification. Anhydrous dichloromethane and benzene used for the reaction were purchased from Kanto Chemical, Japan and were used without further purification.

\section{General procedures for reactions using the Grubbs reagent.}

The Grubbs reagent (20 mol\%) was weighed in the dry box under an Ar atmosphere and the rubber septum was equipped with or without a reflux condenser. A solution of substrate in $\mathrm{CH}_{2} \mathrm{Cl}_{2}$ was introduced into a flask of the Grubbs reagent in one portion. The mixture was kept at the temperature indicated in the table or text for the time indicated. When the reaction was complete, the rubber septum was taken off to expose the reactions mixtures to air under stirring for one hour. The mixture was then directly subjected to column chromatography eluting with an adequate solvent system to afford a crude product. Further purification using column chromatography was carried out to give each pure compound. GC-MS analysis was performed to determine the ratio of the products in each case.

\section{Preparation of 6-t-butyldimethylsilyloxy-1-hexen-3-ol (6).}

To a stirred solution of aldehyde $5(306 \mathrm{mg}, 1.5 \mathrm{mmol})$ in dry THF (15 mL), vinylmagnesium bromide $(0.95 \mathrm{M}, 3.2 \mathrm{~mL}, 3.0 \mathrm{mmol})$ was added at $-78^{\circ} \mathrm{C}$ and the mixture was kept at the same temperature for $1.5 \mathrm{~h}$. Water and sat. ammonium chloride soln. were added and the mixture was extracted with ether. The organic layer was washed with brine, dried $\left(\mathrm{Na}_{2} \mathrm{SO}_{4}\right)$, and was evaporated to afford a residue, which was purified by silica gel column chromatography ( $5 \%$ hexane-EtOAc) to give alcohol 6 (245.5 mg, 70\%); FTIR: 3350, 840, $780 \mathrm{~cm}^{-1} ;{ }^{1} \mathrm{H}-\mathrm{NMR}(300 \mathrm{MHz}) \delta 0.05(6 \mathrm{H}, \mathrm{s}), 0.88(9 \mathrm{H}$, s), $1.61(4 \mathrm{H}, \mathrm{m}), 3.64(2 \mathrm{H}, \mathrm{t}, J=5.7 \mathrm{~Hz}), 4.11(2 \mathrm{H}, \mathrm{m}), 5.07(1 \mathrm{H}, \mathrm{dt}, J=10.5,1.8 \mathrm{~Hz}), 5.22(1 \mathrm{H}, \mathrm{dt}, J$ $=16.2,1.5 \mathrm{~Hz}), 5.85(1 \mathrm{H}, \mathrm{ddd}, J=16.2,10.5,5.7 \mathrm{~Hz}) ;{ }^{13} \mathrm{C} \mathrm{NMR}\left(75 \mathrm{MHz}, \mathrm{CDCl}_{3}\right) \delta-5.4\left(\mathrm{CH}_{3} \times 2\right)$, $18.3(\mathrm{C}), 25.9\left(\mathrm{CH}_{3} \times 3\right), 28.7\left(\mathrm{CH}_{2}\right), 34.4\left(\mathrm{CH}_{2}\right), 63.4\left(\mathrm{CH}_{2}\right), 72.6(\mathrm{CH}), 114.3\left(\mathrm{CH}_{2}\right), 141.1(\mathrm{CH})$; $\mathrm{MS}$ 
(CI) $m / z 231[\mathrm{M}+\mathrm{H}]^{+}, 219,201,161,127,105,81$ (base), 75; HRMS (CI) Found $m / z 231.1777$ $[\mathrm{M}+\mathrm{H}]^{+} \mathrm{C}_{12} \mathrm{H}_{27} \mathrm{O}_{2} \mathrm{Si}$ requires 231.1780 .

Preparation of 6-t-butyldimethylsilyloxy-2-methyl-1-hexen-3-ol (7).

The corresponding Grignard reagent was prepared from 2-bromopropene (1.1 mL, $12.4 \mathrm{mmol})$ and $\mathrm{Mg}(0.3 \mathrm{~g}, 12.4 \mathrm{mmol})$ in dry THF $(10 \mathrm{~mL})$ and then a solution of aldehyde $5(500 \mathrm{mg}, 2.5 \mathrm{mmol})$ in dry THF $(10 \mathrm{~mL})$ was added slowly. The mixture was stirred overnight, and then water and sat. ammonium chloride soln. were added. The mixture was extracted with ether and the organic layer was washed with brine, dried $\left(\mathrm{Na}_{2} \mathrm{SO}_{4}\right)$, and was evaporated to afford a residue. The residue was purified by silica gel column chromatography (10\% hexane-EtOAc) to give alcohol 7 (210.8 $\mathrm{mg}, 35 \%)$; FTIR: 3400, 830, $780 \mathrm{~cm}^{-1} ;{ }^{1} \mathrm{H}-\mathrm{NMR}(300 \mathrm{MHz}) \delta 0.04(6 \mathrm{H}, \mathrm{s}), 0.88(9 \mathrm{H}, \mathrm{s}), 1.60(4 \mathrm{H}, \mathrm{m}), 1.58(3 \mathrm{H}, \mathrm{br} \mathrm{s})$, $2.71(1 \mathrm{H}, \mathrm{br} \mathrm{s}, \mathrm{OH}), 3.64(2 \mathrm{H}, \mathrm{t}, J=6.0 \mathrm{~Hz}), 4.04(1 \mathrm{H}, \mathrm{t}, J=5.4 \mathrm{~Hz}), 4.81(1 \mathrm{H}, \mathrm{br} \mathrm{s}), 4.94(1 \mathrm{H}, \mathrm{br} \mathrm{s})$; ${ }^{13} \mathrm{C}-\mathrm{NMR}(75 \mathrm{MHz}) \delta-5.0\left(\mathrm{CH}_{3} \times 2\right), 18.2\left(\mathrm{CH}_{3}\right), 18.7(\mathrm{C}), 26.3\left(\mathrm{CH}_{3} \times 3\right), 29.2\left(\mathrm{CH}_{2}\right), 32.6\left(\mathrm{CH}_{2}\right), 63.7$ $\left(\mathrm{CH}_{2}\right), 75.7(\mathrm{CH}), 111.1\left(\mathrm{CH}_{2}\right), 147.9(\mathrm{C})$; MS (CI) $\mathrm{m} / z 245[\mathrm{M}+\mathrm{H}]^{+}, 227,202,187,155,145,95$ (base), 75, 67; HRMS (CI) Found $m / z 245.1960[\mathrm{M}+\mathrm{H}]^{+} \mathrm{C}_{13} \mathrm{H}_{29} \mathrm{O}_{2} \mathrm{Si}$ requires 245.1937.

Preparation of (E)-7-t-butyldimethylsilyloxy-2-hepten-4-ol (8).

The Grignard reagent was prepared from trans-1-bromopropene $(0.13 \mathrm{~mL}, 1.49 \mathrm{mmol})$ and $\mathrm{Mg}$ (36.1 mg, $1.5 \mathrm{mmol})$ in dry THF $(10 \mathrm{~mL})$ and a solution of aldehyde $5(100 \mathrm{mg}, 0.5 \mathrm{mmol})$ in dry THF $(5 \mathrm{~mL})$ was added slowly. The mixture was stirred at room temperature for $30 \mathrm{~min}$. Water and sat. ammonium chloride soln. were added and the mixture was extracted with ether. The organic layer was washed with brine, dried $\left(\mathrm{Na}_{2} \mathrm{SO}_{4}\right)$, and was evaporated to afford a residue, which was purified by silica gel column chromatography (2-100 \% hexane-EtOAc) to give alcohol 8 (46.2 $\mathrm{mg}, 38 \%)$; FTIR: $3380 \mathrm{~cm}^{-1}$; ${ }^{1} \mathrm{H}-\mathrm{NMR}(200 \mathrm{MHz}) \delta 0.06(6 \mathrm{H}, \mathrm{s}), 0.89(9 \mathrm{H}, \mathrm{s}), 1.59-1.83(8 \mathrm{H}, \mathrm{m}), 3.61-3.67(3 \mathrm{H}, \mathrm{m})$, 5.36-5.74 (2H, m); ${ }^{13} \mathrm{C}-\mathrm{NMR}(50 \mathrm{MHz}) \delta-5.5\left(\mathrm{CH}_{3} \times 2\right), 17.6\left(\mathrm{CH}_{3}\right), 18.3(\mathrm{C}), 25.9\left(\mathrm{CH}_{3} \times 3\right), 28.9$ $\left(\mathrm{CH}_{2}\right), 34.5\left(\mathrm{CH}_{2}\right), 63.4\left(\mathrm{CH}_{2}\right), 72.7(\mathrm{CH}), 126.4(\mathrm{CH}), 134.3(\mathrm{CH}) ; \mathrm{MS}(\mathrm{CI}) \mathrm{m} / z 245[\mathrm{M}+\mathrm{H}]^{+}, 243$, 227, 211, 187, 169, 145, 111, 95 (base), 75, 67, 57, 41; HRMS (CI) Found $m / z 245.1926[\mathrm{M}+\mathrm{H}]^{+}$ $\mathrm{C}_{13} \mathrm{H}_{29} \mathrm{O}_{2} \mathrm{Si}$ requires 245.1937 .

6-t-butyldimethylsilyloxy-3-hexanone (9); FTIR $1720 \mathrm{~cm}^{-1} ;{ }^{1} \mathrm{H}-\mathrm{NMR}(300 \mathrm{MHz}) \delta 0.01(6 \mathrm{H}, \mathrm{s}), 0.86$ $(9 \mathrm{H}, \mathrm{s}), 1.03(3 \mathrm{H}, \mathrm{t}, J=7.4 \mathrm{~Hz}), 1.76(2 \mathrm{H}$, quint, $J=6.0 \mathrm{~Hz}), 2.41(2 \mathrm{H}, \mathrm{q}, J=6.0 \mathrm{~Hz}), 2.46(2 \mathrm{H}, \mathrm{t}, J=7.4$ $\mathrm{Hz}), 3.59(2 \mathrm{H}, \mathrm{t}, J=6.0 \mathrm{~Hz}) ;{ }^{13} \mathrm{C}-\mathrm{NMR}(75 \mathrm{MHz}) \delta-5.4\left(\mathrm{CH}_{3} \times 2\right), 7.8\left(\mathrm{CH}_{3}\right), 18.3(\mathrm{C}), 25.9\left(\mathrm{CH}_{3} \times 3\right)$, $26.9\left(\mathrm{CH}_{2}\right), 35.9\left(\mathrm{CH}_{2}\right), 38.6\left(\mathrm{CH}_{2}\right), 62.2\left(\mathrm{CH}_{2}\right), 211.5(\mathrm{C}) ; \mathrm{MS}(\mathrm{CI}) \mathrm{m} / z 231[\mathrm{M}+\mathrm{H}]^{+}, 215,173,139$, 99 (base), 75; HRMS (CI) Found $m / z 231.1791[\mathrm{M}+\mathrm{H}]{ }^{+} \mathrm{C}_{12} \mathrm{H}_{27} \mathrm{O}_{2} \mathrm{Si}$ requires 231.1790.

5-t-butyldimethylsilyloxy-2-pentanone (10); FTIR $1720 \mathrm{~cm}^{-1} ;{ }^{1} \mathrm{H}-\mathrm{NMR}(300 \mathrm{MHz}) \delta-0.02(6 \mathrm{H}, \mathrm{s})$, $0.83(9 \mathrm{H}, \mathrm{s}), 1.73(2 \mathrm{H}$, quint, $J=6.0 \mathrm{~Hz}), 2.09(3 \mathrm{H}, \mathrm{s}), 2.45(2 \mathrm{H}, \mathrm{t}, J=6.0 \mathrm{~Hz}), 3.56(2 \mathrm{H}, \mathrm{t}, J=6.0 \mathrm{~Hz})$; 
${ }^{13} \mathrm{C}-\mathrm{NMR}(75 \mathrm{MHz}) \delta-5.4\left(\mathrm{CH}_{3} \times 2\right), 18.2(\mathrm{C}), 25.9\left(\mathrm{CH}_{3} \times 3\right), 26.8\left(\mathrm{CH}_{2}\right), 29.9\left(\mathrm{CH}_{2}\right), 40.0\left(\mathrm{CH}_{3}\right), 62.0$ $\left(\mathrm{CH}_{2}\right), 208.8(\mathrm{C})$; MS (CI) $m / z 217[\mathrm{M}+\mathrm{H}]^{+}, 201,159,125,85$ (base); HRMS (CI) Found $m / z 217.1625$ $[\mathrm{M}+\mathrm{H}]^{+} \mathrm{C}_{11} \mathrm{H}_{25} \mathrm{O} 2 \mathrm{Si}$ requires 217.1624.

7-t-butyldimethylsilyloxy-2-hepten-4-one (11); FTIR 1680, $1640 \mathrm{~cm}^{-1} ;{ }^{1} \mathrm{H}-\mathrm{NMR}(300 \mathrm{MHz}) \delta 0.03(6 \mathrm{H}$, s), $0.88(9 \mathrm{H}, \mathrm{s}), 1.81(2 \mathrm{H}$, quint, $J=6.0 \mathrm{~Hz}), 1.89(3 \mathrm{H}, \mathrm{dd}, J=6.9,1.7 \mathrm{~Hz}), 2.61(2 \mathrm{H}, \mathrm{t}, J=6.0 \mathrm{~Hz}), 3.62$ $(2 \mathrm{H}, \mathrm{t}, J=6.0 \mathrm{~Hz}), 6.12(1 \mathrm{H}, \mathrm{dq}, J=15.9,1.7 \mathrm{~Hz}), 6.85(1 \mathrm{H}, \mathrm{dq}, J=15.9,6.9 \mathrm{~Hz}) ;{ }^{13} \mathrm{C}-\mathrm{NMR}(75 \mathrm{MHz}) \delta$ $-5.3\left(\mathrm{CH}_{3} \times 2\right), 18.2\left(\mathrm{CH}_{3}\right), 18.3(\mathrm{C}), 25.9\left(\mathrm{CH}_{3} \times 3\right), 27.3\left(\mathrm{CH}_{2}\right), 36.2\left(\mathrm{CH}_{2}\right), 62.2\left(\mathrm{CH}_{2}\right), 132.0(\mathrm{CH})$, $142.4(\mathrm{CH}), 200.4(\mathrm{C})$; MS (CI) $m / z 243[\mathrm{M}+\mathrm{H}]^{+}, 228,201,185,151,111$ (base), 89, 75; HRMS (CI) Found $m / z 243.1782[\mathrm{M}+\mathrm{H}]^{+} \mathrm{C}_{13} \mathrm{H}_{27} \mathrm{O}_{2} \mathrm{Si}$ requires 243.1780 .

Preparation of 4-bromo-1-t-butyldimethylsilyloxybutane (13)[8].

To a stirred solution of alcohol $4(2.0 \mathrm{~g}, 9.8 \mathrm{mmol})$ in dichloromethane $(40 \mathrm{~mL})$ was added triethylamine $(1.5 \mathrm{~mL}, 10.8 \mathrm{mmol})$ and methanesulfonyl chloride $(0.83 \mathrm{~mL}, 10.7 \mathrm{mmol})$ at $0^{\circ} \mathrm{C}$. The mixture was stirred for $1 \mathrm{~h}$ and sat. $\mathrm{NaHSO}_{4}$ soln. was added. The mixture was extracted with ether. The organic layer was washed with brine, dried $\left(\mathrm{Na}_{2} \mathrm{SO}_{4}\right)$, and was evaporated to afford mesylate $\mathbf{1 2}$ (2.4 g, 88\%). To a stirred solution of mesylate $12(2.4 \mathrm{~g}, 8.6 \mathrm{mmol})$ in dry THF $(60 \mathrm{~mL})$ was added lithium bromide ( $1.13 \mathrm{~g}, 13.0 \mathrm{mmol})$ and the mixture was refluxed overnight. Sat. $\mathrm{NaHSO}_{4}$ was added and the mixture was extracted with pentane. The organic layer was washed with brine, dried $\left(\mathrm{Na}_{2} \mathrm{SO}_{4}\right)$, and was evaporated to afford a residue, which was purified by silica gel column chromatography (5-100\% hexane-EtOAc) to give bromide $13(1.7 \mathrm{~g}, 66 \%) ;{ }^{1} \mathrm{H}-\mathrm{NMR}(300 \mathrm{MHz}) \delta 0.04(6 \mathrm{H}, \mathrm{s}), 0.89$ $(9 \mathrm{H}, \mathrm{s}), 1.65(2 \mathrm{H}$, quint, $J=6.3 \mathrm{~Hz}), 1.94(2 \mathrm{H}$, quint, $J=6.6 \mathrm{~Hz}), 3.44(2 \mathrm{H}, \mathrm{t}, J=6.6 \mathrm{~Hz}), 3.64(2 \mathrm{H}, \mathrm{t}$, $J=6.3 \mathrm{~Hz}) ;{ }^{13} \mathrm{C}-\mathrm{NMR}(75 \mathrm{MHz}) \delta-5.3\left(\mathrm{CH}_{3} \times 2\right), 18.3(\mathrm{C}), 26.0\left(\mathrm{CH}_{3} \times 3\right), 29.5\left(\mathrm{CH}_{2}\right), 31.3\left(\mathrm{CH}_{2}\right), 34.0$ $\left(\mathrm{CH}_{2}\right), 62.2\left(\mathrm{CH}_{2}\right)$; MS (CI) m/z $267[\mathrm{M}+\mathrm{H}]^{+}, 209,187$ (base), 169, 135, 89; HRMS (CI) Found $\mathrm{m} / z$ $267.0792[\mathrm{M}+\mathrm{H}]^{+} \mathrm{C}_{10} \mathrm{H}_{24} \mathrm{OBrSi}$ requires 267.0780 .

Preparation of 8-t-butyldimethylsilyloxy-2-methyl-2-octen-4-ol (15).

A solution of aldehyde 14 (200 $\mathrm{mg}, 2.38 \mathrm{mmol})$ in dry THF $(5 \mathrm{~mL})$ was added slowly to the Grignard reagent prepared from $13(1.27 \mathrm{~g}, 4.76 \mathrm{mmol})$ and $\mathrm{Mg}(120 \mathrm{mg}, 4.76 \mathrm{mmol})$ in dry THF (5 $\mathrm{mL}$ ). The mixture was stirred overnight at room temperature. Water and sat. ammonium chloride soln. were added and the mixture was extracted with ether. The organic layer was washed with brine, dried $\left(\mathrm{Na}_{2} \mathrm{SO}_{4}\right)$, and was evaporated to afford a residue, which was purified by silica gel column chromatography (5-100 \% hexane-EtOAc) to give alcohol 15 (399 mg, 62\%); FTIR: 3350, 840, 780 $\mathrm{cm}^{-1} ;{ }^{1} \mathrm{H}-\mathrm{NMR}(300 \mathrm{MHz}) \delta 0.02(6 \mathrm{H}, \mathrm{s}), 0.86(9 \mathrm{H}, \mathrm{s}), 1.40(6 \mathrm{H}, \mathrm{m}), 1.65(3 \mathrm{H}, \mathrm{d}, J=1.5 \mathrm{~Hz}), 1.69(3 \mathrm{H}$, d, $J=1.2 \mathrm{~Hz}), 3.58(2 \mathrm{H}, \mathrm{t}, J=6.6 \mathrm{~Hz}), 4.31(1 \mathrm{H}, \mathrm{dt}, J=8.7,6.6 \mathrm{~Hz}),), 5.12(1 \mathrm{H}, \mathrm{br} \mathrm{d}, J=8.7 \mathrm{~Hz})$; ${ }^{13} \mathrm{C}-\mathrm{NMR}(75 \mathrm{MHz}) \delta-5.3\left(\mathrm{CH}_{3} \times 2\right), 18.2\left(\mathrm{CH}_{3}\right), 18.3(\mathrm{C}), 21.7\left(\mathrm{CH}_{2}\right), 25.8\left(\mathrm{CH}_{3}\right), 25.9\left(\mathrm{CH}_{3} \times 3\right), 32.7$ $\left(\mathrm{CH}_{2}\right), 37.4\left(\mathrm{CH}_{2}\right), 63.1\left(\mathrm{CH}_{2}\right), 68.6(\mathrm{CH}), 128.2(\mathrm{CH}), 134.8(\mathrm{C}) ; \mathrm{MS}(\mathrm{CI}) \mathrm{m} / z 272[\mathrm{M}-\mathrm{H}+\mathrm{H}]^{+}, 255$, 
215, 197, 139, 123 (base), 85, 81; HRMS (CI) Found $m / z 272.2167[\mathrm{M}-\mathrm{H}+\mathrm{H}]^{+} \mathrm{C}_{15} \mathrm{H}_{32} \mathrm{O}_{2} \mathrm{Si}$ requires 272.2172 .

8-t-butyldimethylsilyloxy-2-methyl-2-octen-4-ol (16); FTIR: $1690 \mathrm{~cm}^{-1}$; ${ }^{1} \mathrm{H}-\mathrm{NMR}(200 \mathrm{MHz}) \delta 0.04$ $(6 \mathrm{H}, \mathrm{s}), 0.89(9 \mathrm{H}, \mathrm{s}), 1.57(5 \mathrm{H}, \mathrm{s}), 1.70(2 \mathrm{H}, \mathrm{dd}, J=6.4,1.2 \mathrm{~Hz}), 1.88(2 \mathrm{H}, \mathrm{d}, J=1.2 \mathrm{~Hz}), 2.14(2 \mathrm{H}, \mathrm{d}$, $J=1.2 \mathrm{~Hz}), 2.42(1 \mathrm{H}, \mathrm{t}, J=6.8 \mathrm{~Hz}), 3.61(2 \mathrm{H}, \mathrm{t}, J=6.4 \mathrm{~Hz}), 6.07(1 \mathrm{H}, \mathrm{t}, J=1.4 \mathrm{~Hz}) ;{ }^{13} \mathrm{C}-\mathrm{NMR}(50 \mathrm{MHz}) \delta$ -5.3 $\left(\mathrm{CH}_{3} \times 2\right), 10.0,18.4(\mathrm{C}), 20.7,26.0\left(\mathrm{CH}_{3} \times 3\right), 27.6,32.4,44.0,63.0,123.8$; MS (CI) $\mathrm{m} / z 271$ $[\mathrm{M}+\mathrm{H}]^{+}, 255,239,213,197,171,155,139$ (base), 123, 101, 95, 83, 75, 67, 59, 41; HRMS (CI) Found $m / z 271.2105[\mathrm{M}+\mathrm{H}]^{+} \mathrm{C}_{15} \mathrm{H}_{31} \mathrm{O}_{2} \mathrm{Si}$ requires 271.2093.

8-t-butyldimethylsilyloxy-2-methyl-1,3-octadiene (17); ${ }^{1} \mathrm{H}-\mathrm{NMR}(200 \mathrm{MHz}) \delta 0.05(6 \mathrm{H}, \mathrm{s}), 0.90(9 \mathrm{H}$, s), 1.14-1.83 (9H, m), $2.13(2 \mathrm{H}, \mathrm{q}, J=6.8 \mathrm{~Hz}), 4.86(2 \mathrm{H}, \mathrm{s}), 5.58-5.72(1 \mathrm{H}, \mathrm{m}), 6.14(1 \mathrm{H}, \mathrm{d}, J=15.6$ $\mathrm{Hz}),{ }^{13} \mathrm{C}-\mathrm{NMR}(50 \mathrm{MHz}) \delta-5.3\left(\mathrm{CH}_{3} \times 2\right), 18.4(\mathrm{C}), 18.7\left(\mathrm{CH}_{2}\right), 21.7\left(\mathrm{CH}_{3}\right), 25.6\left(\mathrm{CH}_{2}\right), 26.0\left(\mathrm{CH}_{3} \times\right.$ 3), $32.4\left(\mathrm{CH}_{2}\right), 63.1\left(\mathrm{CH}_{2}\right), 114.2\left(\mathrm{CH}_{2}\right), 130.8(\mathrm{CH}), 133.0(\mathrm{CH}), 142.2(\mathrm{C})$; $\mathrm{MS}(\mathrm{CI}) \mathrm{m} / z 255[\mathrm{M}+\mathrm{H}]^{+}$, 239, 211, 197, 155, 139, 123(base), 95, 83, 75, 67, 41; HRMS (CI) Found $m / z 255.2136[\mathrm{M}+\mathrm{H}]^{+}$ $\mathrm{C}_{15} \mathrm{H}_{31} \mathrm{OSi}$ requires 255.2144 .

\section{References}

1. Nakashima, K.; Ito, R.; Sono, M.; Tori, M. Heterocycles 2000, 53, 301-314.

2. Hoye, T. R.; Zhao, H. Org. Lett. 1999, 1, 169-172.

3. Backvall, J. -E.; Andreasson, U.; Tetrahedron Lett. 1993, 34, 5459-5462.

4. McGrath, D. V.; Grubbs, R. H. Organometallics 1994, 13, 224-235

5. Cadot, C.; Dalko, P. I.; Cossy, J. Tetrahedron Lett. 2002, 43, 1839-1841.

6. Danishefsky, S. J.; Pearson, W. H. J. Org. Chem. 1983, 48, 3865-3866.

7. Branchaud, B. P. J. Org. Chem. 1983, 48, 3531-3538.

8. Tauch, P.; Fallis, A. G. J. Org. Chem. 1999, 64, 6960-6968.

Sample availability: Samples are not available.

(C) 2004 by MDPI (http://www.mdpi.org). Reproduction is permitted for noncommercial purposes. 